\title{
Detection of relevant extracardiac findings on coronary computed tomography angiography vs. invasive coronary angiography
}

\author{
Dominik Laskowski ${ }^{1} \cdot$ Sarah Feger ${ }^{1} \cdot$ Maria Bosserdt $^{1} \cdot$ Elke Zimmermann $^{1} \cdot$ Mahmoud Mohamed $^{1}$.

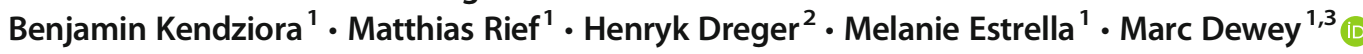

Received: 20 October 2020 / Revised: 11 March 2021 / Accepted: 31 March 2021 / Published online: 15 June 2021

(C) The Author(s) 2021

\begin{abstract}
Objectives To compare the detection of relevant extracardiac findings (ECFs) on coronary computed tomography angiography (CTA) and invasive coronary angiography (ICA) and evaluate the potential clinical benefit of their detection.

Methods This is the prespecified subanalysis of ECFs in patients presenting with a clinical indication for ICA based on atypical angina and suspected coronary artery disease (CAD) included in the prospective single-center randomized controlled Coronary Artery Disease Management (CAD-Man) study. ECFs requiring immediate therapy and/or further workup including additional imaging were defined as clinically relevant. We evaluated the scope of ECFs in 329 patients and analyzed the potential clinical benefit of their detection.

Results ECFs were detected in 107 of 329 patients (32.5\%; CTA: 101/167, 60.5\%; ICA: 6/162, 3.7\%; $p<.001)$. Fifty-nine patients had clinically relevant ECFs (17.9\%; CTA: 55/167, 32.9\%; ICA: 4/162, 2.5\%; $p<.001)$. In the CTA group, ECFs potentially explained atypical chest pain in 13 of 101 patients with ECFs (12.9\%). After initiation of therapy, chest pain improved in $4(4.0 \%)$ and resolved in 7 patients (6.9\%). Follow-up imaging was recommended in 33 (10.0\%; CTA: 30/167, 18.0\%; ICA: $3 / 162,1.9 \%)$ and additional clinic consultation in 26 patients (7.9\%; CTA: 25/167, 15.0\%; ICA: 1/162, 0.6\%). Malignancy was newly diagnosed in one patient $(0.3 \%$; CTA: $1 / 167,0.6 \%$; ICA: 0$)$.

Conclusions In this randomized study, CTA but not ICA detected clinically relevant ECFs that may point to possible other causes of chest pain in patients without CAD. Thus, CTA might preclude the need for ICA in those patients.

Trial registration NCT Unique ID: 00844220

Key Points

- CTA detects ten times more clinically relevant ECFs than ICA.

- Actionable clinically relevant ECFs affect patient management and therapy and may thus improve chest pain.

- Detection of ECFs explaining chest pain on CTA might preclude the need for performing ICA.
\end{abstract}

Keywords Computed tomography angiography $\cdot$ Coronary artery disease $\cdot$ Coronary angiography $\cdot$ Chest pain $\cdot$ Incidental findings

Abbreviations

CAD Coronary artery disease

Marc Dewey

marc.dewey@charite.de

1 Department of Radiology, Charité - Universitätsmedizin Berlin, Charitéplatz 1, 10117 Berlin, Germany

2 Department of Cardiology, Charité - Universitätsmedizin Berlin, Charitéplatz 1, 10117 Berlin, Germany

3 Berlin Institute of Health and DZHK (German Centre for Cardiovascular Research) Partner Site, Berlin, Germany
CI Confidence interval

CTA Coronary computed tomography angiography

ECF Extracardiac finding

FOV Field of view

ICA Invasive coronary angiography

\section{Introduction}

Coronary computed tomography angiography (CTA) and invasive coronary angiography (ICA) are both well-established methods for the assessment of cardiac and coronary anatomy 
and pathology. However, CTA is a radiological, noninvasive technique whereas ICA is a cardiological, invasive method. While both CTA and ICA also visualize surrounding structures, such as lungs, mediastinum, and chest wall, CTA is clearly superior to ICA in this respect. Detailed evaluation of adjacent anatomy allows identification of extracardiac findings (ECFs) which may potentially explain the patient's symptoms or require further workup and therapy. This may be especially important for patients in whom cardiac pathologies have been ruled out, but chest pain still persists. Furthermore, patients in whom CTA rules out CAD while simultaneously detecting ECFs explaining chest pain might be spared an ICA. Previous studies show that ECFs are common in patients undergoing CTA [1-18]. On the other hand, there is an ongoing debate in the scientific community on the need to look for ECFs in CTA scans $[19,20]$. At the same time, results on the clinical relevance of ECFs vary strongly from one study to the next, whereas robust evidence regarding the potential clinical benefit of detecting such findings with long-term follow-up is virtually not existing. We found only one study on the follow-up assessment of ECFs in patients with chest pain. In this study, some of the ECFs were identified as treatable causes of the patients' chest pain [2].

The Coronary Artery Disease Management (CAD-Man) prospective randomized controlled trial has already shown that CTA performed instead of ICA in patients with atypical angina and a low to intermediate CAD risk significantly enhances the diagnostic yield of coronary angiography, reduces minor procedural complications, and shortens hospitalization compared with direct ICA [21]. The primary aim of this substudy was to analyze the potential clinical benefit of the identification of clinically relevant ECFs on CTA and ICA in patients presenting with atypical chest pain by assessing the scope of recommended diagnostic procedures and therapeutic consequences following the detection of such ECFs. The ancillary aim of our substudy was to investigate the impact of patient age, sex, and smoking behavior on the prevalence of detected ECFs.

\section{Materials and methods}

\section{Study design and participants}

We performed a subanalysis of ECFs based on data from the prospective single-center randomized controlled Coronary Artery Disease Management (CAD-Man) study, in which the protocol included ECFs as a prospectively collected item with clear management recommendations to patients based on predefined findings [21]. Recruitment of study participants has been described in detail elsewhere [21]. Briefly, between February 18, 2009, and August 27, 2015, we enrolled 340 patients with atypical angina or chest pain referred for ICA due to suspected CAD. The patients were randomly assigned to CTA (168/340) and ICA (172/340). Atypical angina was defined as the appearance of no more than two of the three criteria for typical angina pectoris, which are retrosternal chest pain, precipitation of pain by exertion, and rapid relief of symptoms at rest or within $30 \mathrm{~s}$ to 10 min following nitroglycerine administration [22].

\section{CT and ICA procedure}

CT examinations were performed preferably in the early morning when individuals have lower heart rates. A contrast agent with an iodine concentration of $350 \mathrm{mg} / \mathrm{ml}$ (Iobitridol, Xenetix ${ }^{\circledR} 350$, Guerbet) was used. Patients in the ICA group underwent diagnostic testing after hospital admission according to clinical practice at our institution, which follows European guidelines [23]. The same contrast agent as for CTA was used. ICA examinations were evaluated independently by at least two board-certified cardiologists with a minimum of 5 years of experience following local standards of care. Radiation dose was assessed in both study groups for initial diagnostic procedure. Details of the calculation method were described elsewhere [21].

\section{CT image acquisition and reading}

CTA was performed on one of two 320-row CT scanners

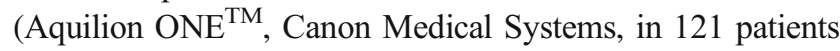
and Aquilion ONE ViSION Edition in 44 patients). Two patients assigned to the CTA group underwent ICA based on the clinician's decision, leaving 165 patients who underwent CTA. Standard soft tissue and lung reconstructions of the raw data with $3-5-\mathrm{mm}$ slice thickness on a large $320-\mathrm{mm}$ FOV with the center of the reconstruction window at 75 $80 \%$ of the RR interval were generated for assessment of noncardiac structures. All CT images were independently reviewed for ECFs by two readers, at least one of them a board-certified radiologist with a minimum of 5 years of experience. The final decisions were made by consensus. Clinically relevant ECFs were reported to the patient's clinical physician, followed by immediate further management if required.

\section{Assessment of ECFs}

An ECF was defined as an abnormality that was detected on CTA or ICA scans and located outside the heart and pericardium. First, we distinguished clinically relevant and nonsignificant ECFs. Categorization of ECFs was largely based on the classification proposed by Karius et al [24]. Clinically relevant ECFs were defined as requiring immediate therapy and/or further workup including 
additional imaging [24]. All other ECFs were classified as clinically nonsignificant. In the set of clinically relevant ECFs, we further tried to identify acutely life-threatening and malignant ECFs. Second, we quantified the diagnostic actions recommended for ECFs and analyzed whether they were followed by patients and if they had any therapeutic consequences. A therapeutic consequence was assumed if the patient received particular treatment for a clinically relevant ECF. Third, we evaluated if ECFs could potentially explain atypical chest pain. Based on theoretical considerations, we sought for alternative conditions that could account for a patient's symptoms such as pneumonia, hiatal hernia, or cancer. All ECFs were assigned to one of five anatomical categories (lungs, upper abdomen, bones, vessels, and mediastinum) for quantitative analysis of their anatomical sites and distribution. The remaining ECFs were assigned to the category of "other adjacent regions". Upon request of the editor in chief, we supplemented this prespecified analysis by an analysis of the potential of ECFs to explain the patient's chest pain. Furthermore, we analyzed a potential association between the number of detected ECFs and patient age, sex, and smoking behavior defined as prior or current smoking.

\section{Follow-up of ECFs}

Long-term follow-up for a median of 3.75 years was available. Follow-up data were available from follow-up questionnaires completed by the study participants. The patients were contacted by phone if some responses were missing or unclear. Additional follow-up data were available from the patients' electronic medical record.

\section{Statistical analysis}

All statistical analyses were performed with SAS University Edition 9.4 software. Categorical variables are presented as percentage (\%) and continuous normally distributed variables as mean and standard deviation, while not normally distributed continuous outcomes are presented as median and interquartile range. We used Fisher's exact test to compare presence of the ECFs between the two groups. Both outcomes (clinically relevant and nonsignificant) met the Poisson distribution ( $p=0.838, p=0.32$, respectively); nevertheless, variance was larger than the mean for both outcomes. Consequently, univariate analysis of the number of detected ECFs and each independent variable (patient age, sex, and smoking behavior) was performed by negative binomial regression. A $p$-value $<0.025$ was considered to indicate a statistically significant difference in multivariate negative binomial regression analysis.

\section{Results}

\section{Detection of relevant ECFs}

Baseline patient characteristics in the CTA and ICA groups are presented in ESM Table 1. One of 168 patients in the CTA group and 10 of 172 patients in the ICA group withdrew informed consent and did not undergo the assigned procedure. Consequently, 329 patients were available for analysis. The median exposure to radiation was $4.8 \mathrm{mSv}$ (interquartile range: 4.1-5.8) in the CTA group and $6.0 \mathrm{mSv}$ in the ICA group (3.0-10.0). Seventy-nine clinically relevant ECFs were detected in 59 patients $(59 / 329,17.9 \%$; CTA: $55 / 167,32.9 \%$; ICA: $4 / 162,2.5 \%$ ). In 43 patients (13.1\%; CTA: 40, ICA: 3 ), one clinically relevant ECF was present, whereas 16 patients (4.9\%; CTA: 15, ICA: 1 ) had two or more clinically relevant ECFs. Overall, ECFs were found in 107 of 329 patients $(32.5 \%)$, among them 101 in the CTA and only six in the ICA group (CTA: 101/167, 60.5\%; ICA: 6/162, 3.7\%; $p<$ .001) (Fig. 1). These 107 patients had a total of 186 ECFs. Details of the distribution and frequencies of ECFs by group (CTA versus ICA) and anatomy are given in ESM Table 2. The distribution of clinically relevant ECFs by anatomical region is shown in Fig. 2. The most common ECFs were hiatal hernia $(30.3 \%)$, suspicious pulmonary nodules $(13.9 \%)$, and liver abnormalities (11.4\%).

\section{ECFs as a possible cause of chest pain}

Among the 329 patients available for analysis, 144 had atypical angina, 177 nonanginal chest pain, and 8 had other chest discomfort. In the CTA group, we detected ECFs that could potentially explain atypical chest pain in 13 of 101 patients with ECFs (12.9\%). Moreover, in all 13 of those patients, CTA ruled out significant CAD (coronary stenosis $>50 \%$ ), precluding the need for ICA. Thus, the potential risk associated to ICA might have been avoided and dedicated therapy initiated instead. That is a definite benefit for patients undergoing CTA. Conversely, in the ICA group, none of the ECFs could explain chest pain. All ECFs that required further imaging, consultation, or therapy were immediately reported to the patient and patient's clinical physician. All recommendations were followed. Hiatal hernia (Fig. 3) was the most common ECF potentially causing chest pain (Table 1). At the time of the last follow-up performed after initiation of treatment in the 13 patients with ECFs potentially explaining symptoms, chest pain was improved in four patients $(4.0 \%$ of those with ECFs on CTA) and resolved in seven patients (6.9\%), leaving only two patients who still had chest pain. We did not detect any acutely life-threatening ECFs such as pulmonary embolism in either the CTA or the ICA group. Conversely, five malignant ECFs were detected in three patients $(3 / 59 ; 5.1 \%)$, all of them 
Fig. 1 Prevalence of incidental ECFs in the CTA and ICA groups of our study

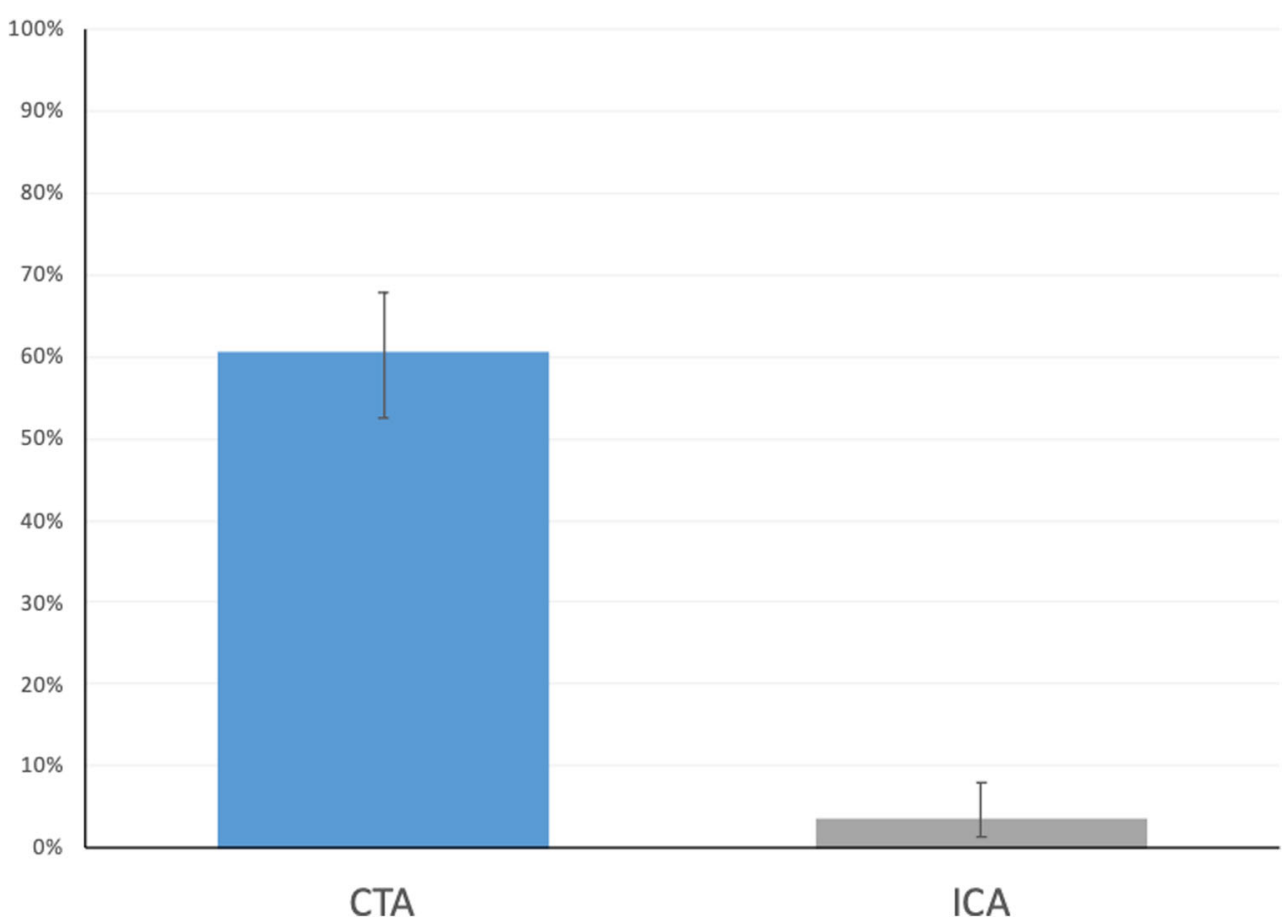

in the CTA group. Malignancies were most commonly located in the lung, followed by the mediastinum and liver (Table 2).

\section{Recommendations and management of ECFs}

Further imaging for workup of detected ECFs was recommended in 33 patients (10.0\%; CTA: 30/167, 18.0\%; ICA: $3 / 162,1.9 \%)$. The most frequent follow-up imaging test was chest CT followed by abdominal ultrasonography (Table 3). Additional clinical consultations by a specialist were recommended in 26 patients (7.9\%; CTA: 25/167, 15.0\%; ICA: $1 / 162,0.6 \%)$. The most frequently recommended specialty consultation was a gastrointestinal consultation followed by an orthopedic consultation (Table 3 ). Thus, a total of 72 diagnostic procedures (38 further imaging examinations, 34 clinical consultations) were recommended in 59 patients (CTA: 55; ICA: 4). Most patients followed the recommendations given based on their ECFs $(49 / 59 ; 83.1 \%)$. In the CTA group, 10 patients $(10 / 59 ; 16.9 \%)$ did not undergo the recommended diagnostic procedures. None of the patients in our study had adverse events related to the recommended additional test procedures or treatment.

Patients received appropriate therapy based on detected ECFs if required. In 17 patients, the clinically relevant ECFs had therapeutic consequences (17/329, 5.2\%; CTA group: 16/ $167,9.6 \%$, ICA group: 1/162, 0.6\%) (Table 4). In 12 patients in the CTA group $(12 / 17 ; 70.6 \%)$, the therapeutic consequence was medical therapy (antacid medication in 9 patients,
Fig. 2 Distribution of clinically relevant ECFs by anatomical region

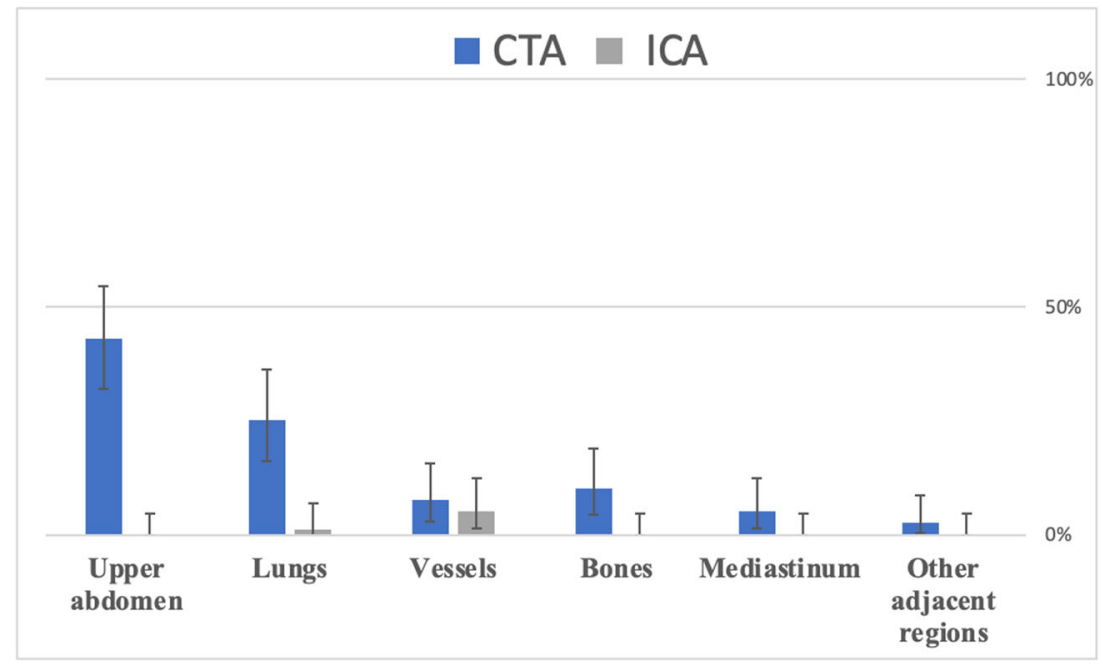



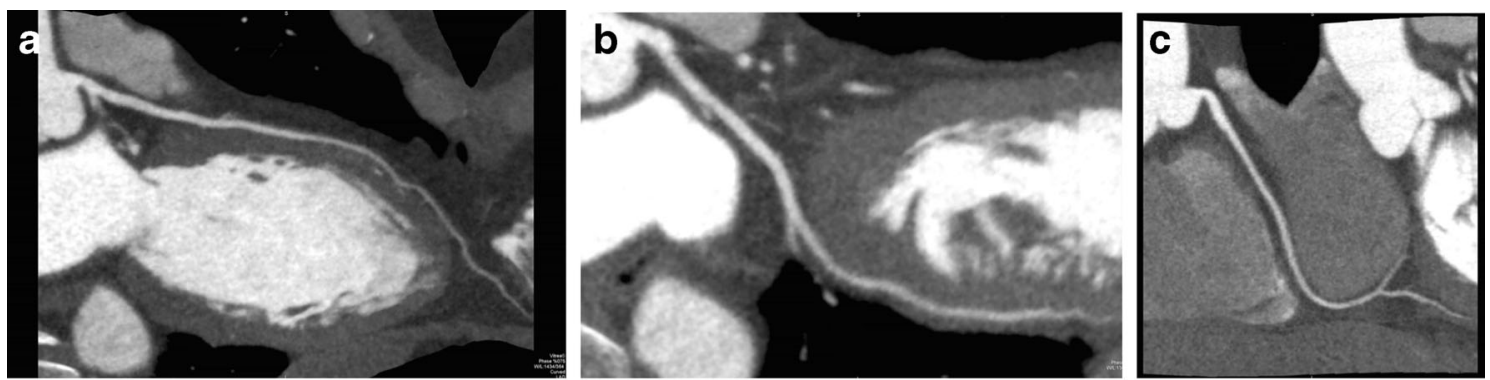

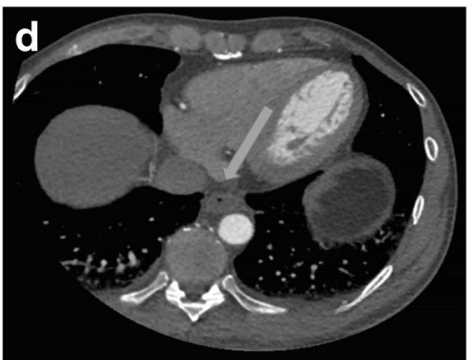

Fig. 3 Forty-eight-year-old man with a $3.7 \times 4.0 \mathrm{~cm}$ esophageal hiatal hernia (arrow), which after gastroenterological consultation and initiation of acid blocker treatment turned out to be a potential cause of chest pain in this patient. CTA detected no significant coronary artery stenoses in this

antibiotics in 2 patients, and antihypertensive in 1 patient). Two patients each in the CTA group $(2 / 17 ; 11.8 \%)$ underwent physical therapy and surgery (thoracotomy for lung cancer and surgery of upside-down stomach). The case of newly diagnosed lung cancer is presented in Fig. 4. This patient had curative thoracic surgery and chemoradiotherapy and has since been free of recurrence for almost 5 years. The one patient with therapeutic consequences for ECFs in the ICA group was newly diagnosed with pulmonary hypertension. As a result of this ECF, the planned aortic valve replacement therapy had to be canceled. Table 4 summarizes clinically relevant ECFs by anatomical territory and in relation to

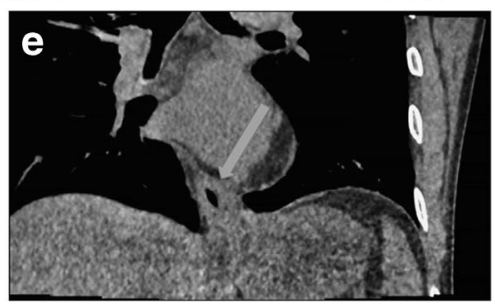

patient. a LMA and LAD, curved reconstruction. b LCX, curved reconstruction. c RCA curved reconstruction. d Soft tissue reconstruction, axial plane. e Soft tissue reconstruction, coronal plane

randomized group and gives information if ECFs had therapeutic consequences.

\section{Impact of variables on prevalence of ECFs}

We analyzed the influence of patient age, sex, and smoking behavior on the prevalence of ECFs, separately for all, clinically relevant, and clinically nonsignificant ECFs. The results of multivariate analysis of potential associations between any of the three variables and category of ECF are presented in Table 5. A statistically significant positive association was revealed between female sex and all ECFs as well as clinically
Table 1 ECFs potentially explaining chest pain

\begin{tabular}{|c|c|c|c|c|c|c|}
\hline \multirow[t]{2}{*}{ ECFs potentially causing chest pain } & \multirow[t]{2}{*}{ Absolute number } & \multicolumn{2}{|c|}{ Study group } & \multicolumn{2}{|c|}{$\begin{array}{l}\text { Diagnosis of } \\
\text { significant CAD }\end{array}$} & \multirow[t]{2}{*}{ Prevalence } \\
\hline & & CTA & ICA & Yes & No & \\
\hline \multicolumn{7}{|l|}{ Upper abdomen } \\
\hline Hiatal hernia & 7 & 7 & 0 & 0 & 7 & $2.1 \%$ \\
\hline \multicolumn{7}{|l|}{ Bones } \\
\hline Spinal degeneration & 2 & 2 & 0 & 0 & 2 & $0.6 \%$ \\
\hline Forestier disease & 1 & 1 & 0 & 0 & 1 & $0.3 \%$ \\
\hline \multicolumn{7}{|l|}{ Lungs } \\
\hline Pneumonia & 2 & 2 & 0 & 0 & 2 & $0.6 \%$ \\
\hline Lung cancer & 1 & 1 & 0 & 0 & 1 & $0.3 \%$ \\
\hline $\begin{array}{l}\text { No. of patients with ECFs } \\
\text { potentially explaining chest pain }\end{array}$ & 13 & 13 & 0 & 0 & 13 & $4.0 \%$ \\
\hline
\end{tabular}

There was only one extracardiac finding potentially leading to chest pain in each patient $E C F$ extracardiac finding, CTA coronary computed tomography angiography, ICA invasive coronary angiography 
Table 2 Malignant incidental ECFs

\begin{tabular}{lll}
\hline Malignant ECFs & CTA & ICA \\
\hline Newly diagnosed & & \\
$\quad$ Lung cancer & 1 & 0 \\
Previously known & & \\
$\quad$ Lung metastasis & 2 & 0 \\
Liver metastasis & 1 & 0 \\
Mediastinal metastasis & 1 & 0 \\
Total no. of malignant ECFs & 5 & 0 \\
Total no. of patients with malignant ECFs & 4 & 0 \\
Frequency in relation to all clinically relevant ECFs in respective group & $6.8 \%$ & $0 \%$ \\
Frequency in relation to patients with clinically relevant ECFs in respective group & $5.5 \%$ & $0 \%$ \\
\hline
\end{tabular}

$E C F$ extracardiac finding, CTA coronary computed tomography angiography, ICA invasive coronary angiography relevant ECFs. Women were more likely to have clinically relevant ECFs than men (OR, 1.65; 95\% CI 1.08-2.51; $p=$ 0.019). For patient age, there was no significant correlation between increasing age and prevalence of ECFs. The same influence was observed regarding smoking behavior.

\section{Discussion}

In this study, we performed systematic long-term follow-up for a median of 3.75 years of initially suspicious ECFs found on CTA and ICA in patients with atypical chest pain and suspected CAD who participated in our randomized trial. Thus, we could evaluate the potential benefit of detection of relevant ECFs and analyze the consequences of the different detection rates of ECFs on CTA vs. ICA. Our study shows that the higher detection rate of ECFs by CTA is clinically beneficial as the findings include potential differential diagnoses that explain chest pain in patients in whom the diagnostic procedure rules out significant CAD. This could eliminate the need to perform ICA in such cases, which is invasive and associated with more procedural complications than CTA. Finally, detection of relevant ECFs results in more diagnostic procedures. While this may be associated with potential procedural complications and increase healthcare costs, it can improve quality of life of affected patients.

Two studies performed so far reported clinically relevant ECFs on CTA as a possible underlying cause of chest pain when CAD was ruled out by the procedure [1,2]. Karius et al [1] investigated 2330 patients with chest pain, identifying 7.9\% ECFs that might explain their pain. In a study population of 1778 patients, Williams et al [2] identified ECFs that were then assessed as possible alternative causes of chest pain in $3 \%$ of cases. In our study, $4.0 \%$ of patients were diagnosed with ECFs on CTA which could cause anginal symptoms. Following initiation of treatment in this subgroup, chest pain was improved in $4 \%$ and resolved in $7 \%$ of all patients in whom ECFs were detected by CTA. The most common
Table 3 Frequency of recommended follow-up imaging investigations and clinic consultations based on detected ECFs on CTA vs. ICA

\begin{tabular}{llllll}
\hline \multirow{2}{*}{ Follow-up imaging } & \multicolumn{2}{l}{ Frequency } & & Follow-up clinic consultation & \multicolumn{2}{c}{ Frequency } \\
\cline { 2 - 3 } \cline { 2 - 5 } & CTA & ICA & & CTA & ICA \\
\hline Chest CT & 17 & 1 & Gastrointestinal & 23 & 0 \\
Abdominal ultrasonography & 8 & 0 & Orthopedic & 7 & 0 \\
Echocardiography & 4 & 2 & Pulmonary & 3 & 1 \\
Mammography & 2 & 0 & & & \\
Cardiac MRI & 1 & 0 & & & \\
PET/CT & 1 & 0 & & & \\
Chest plain radiography & 1 & 0 & & 33 & 1 \\
Thoracic spine MRI & 1 & 0 & & & \\
Total number of procedures & 35 & 3 & & & \\
\hline
\end{tabular}

$E C F$ extracardiac finding, $C T A$ coronary computed tomography angiography, ICA invasive coronary angiography 
Table 4 Clinically relevant incidental ECFs by anatomical region and therapeutic consequences.

\begin{tabular}{|c|c|c|c|c|}
\hline \multirow[t]{3}{*}{ ECFs by anatomical region } & \multirow{2}{*}{\multicolumn{2}{|c|}{$\begin{array}{l}\text { Coronary computed tomography angiography } \\
\text { (CTA) } \\
\text { Therapeutic consequences }\end{array}$}} & \multicolumn{2}{|c|}{$\begin{array}{l}\text { Invasive coronary angiography } \\
\text { (ICA) }\end{array}$} \\
\hline & & & & \\
\hline & No $(n=58)$ & Yes $(n=16)$ & No $(n=4)$ & Yes $(n=1)$ \\
\hline \multicolumn{5}{|l|}{ Upper abdomen $(n=34)$} \\
\hline Hiatal hernia & 14 & 10 & & \\
\hline Liver hemangioma/mass/cystic lesion & 8 & & & \\
\hline Malignancy & 1 & & & \\
\hline Adrenal mass & 1 & & & \\
\hline \multicolumn{5}{|l|}{ Lungs $(n=21)$} \\
\hline Suspicious pulmonary nodules & 11 & & & \\
\hline Malignancy & 2 & 1 & & \\
\hline Chronic changes of lung parenchyma and bronchial system & 2 & & & \\
\hline Pulmonary infiltration & & 2 & & \\
\hline Pulmonary hypertension & & 1 & & 1 \\
\hline Pleural effusion & 1 & & & \\
\hline \multicolumn{5}{|l|}{ Vessels $(n=10)$} \\
\hline Aortic aneurysm & 2 & & 1 & \\
\hline Aortic stenosis & & & 2 & \\
\hline Dilatation of pulmonary arteries & 1 & & & \\
\hline Other abnormalities of aorta & 3 & & 1 & \\
\hline \multicolumn{5}{|l|}{ Bones $(n=8)$} \\
\hline Spinal degeneration/destruction & 4 & 2 & & \\
\hline Forestier disease & 2 & & & \\
\hline \multicolumn{5}{|l|}{ Mediastinum $(n=4)$} \\
\hline Enlarged lymph node & 1 & & & \\
\hline Mediastinal mass & 1 & & & \\
\hline Mediastinal malignancy & 1 & & & \\
\hline Thymus hyperplasia & 1 & & & \\
\hline \multicolumn{5}{|l|}{ Other adjacent regions $(n=2)$} \\
\hline Breast lesion & 2 & & & \\
\hline
\end{tabular}

Therapeutic consequences were assumed to be present if the patient received particular treatment aimed at the clinically relevant ECF $E C F$ extracardiac finding, CTA coronary computed tomography angiography, ICA invasive coronary angiography
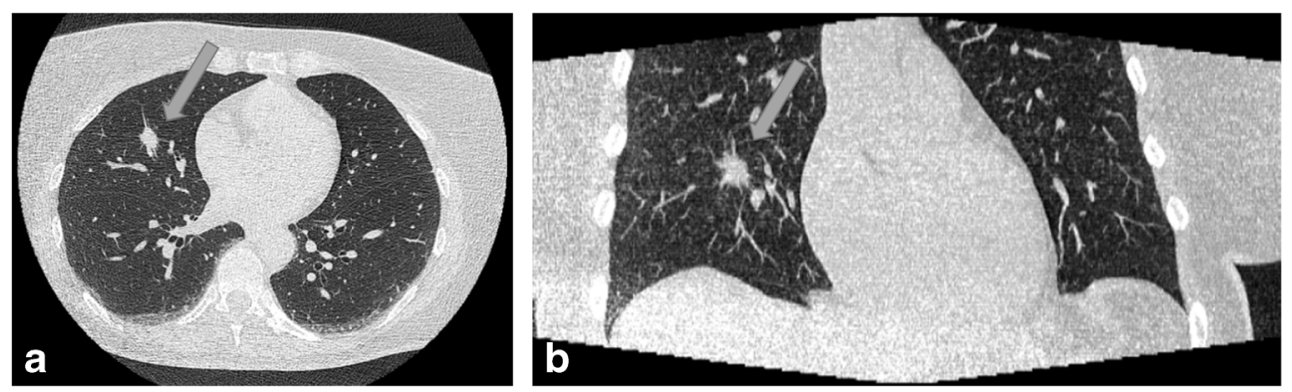

Fig. 4 Incidentally detected poorly differentiated acinar adenocarcinoma of the lung in a 61-year-old woman with atypical chest pain. a Spiculated $9 \times 14 \mathrm{~mm}$ consolidation in segment 5 of the right lung in CTA (arrow), axial plane. b CTA, coronal plane. c Subsequent PET-CT examination confirms lung cancer (arrow) 
Table 5 Associations between risk variables and the presence of incidental ECFs

\begin{tabular}{|c|c|c|c|c|c|c|}
\hline \multirow[t]{2}{*}{ Risk variable } & \multicolumn{2}{|l|}{ All ECFs } & \multicolumn{2}{|c|}{ Clinically nonsignificant ECFs } & \multicolumn{2}{|c|}{ Clinically relevant ECFs } \\
\hline & Odds ratio $(\mathrm{CI})$ & $p$ value & Odds ratio $(\mathrm{CI})$ & $p$ value & Odds ratio $(\mathrm{CI})$ & $p$ value \\
\hline Female gender & $1.38(1.007-1.886)$ & 0.045 & $1.08(0.673-1.742)$ & 0.74 & $1.652(1.08-2.512)$ & 0.019 \\
\hline Male gender & $0.725(0.530-0.993)$ & 0.045 & $0.923(0.574-1.484)$ & 0.74 & $0.605(0.398-0.920)$ & 0.019 \\
\hline Smoking & $1.202(0.887-1.628)$ & 0.235 & $1.353(0.847-2.162)$ & 0.206 & $1.103(0.741-1.644)$ & 0.629 \\
\hline Age $>57$ years & $1.196(0.863-1.659)$ & 0.282 & $1.469(0.877-2.462)$ & 0.144 & $1.035(0.678-1.580)$ & 0.874 \\
\hline
\end{tabular}

$C I$ confidence interval, ECF extracardiac finding

findings were hiatal hernia (Fig. 3) and spinal abnormalities such as Forestier disease (Fig. 5), similar to the study of Karius et al. The use of large FOV resulted in higher detection of ECFs [7]. The novelty of our study is that we performed a long-term analysis of clinical consequences of relevant ECFs in patients with atypical chest pain and suspected CAD randomly assigned to CTA or ICA. We systematically followed up detected ECFs over 3 years to analyze the potential clinical benefit of their detection for patients.

Obviously, the detection of ECFs leads to more diagnostic procedure with potential complications, especially when an invasive procedure is needed, such as a lung biopsy. However, the vast majority of recommended follow-up procedures were noninvasive diagnostic imaging tests such as chest CT and abdominal ultrasonography. Nonetheless, in our study, none of the patients had adverse events related to the recommended diagnostic procedures. Neither Williams et al nor Bendix et al, who both investigated patients with chest pain, reported any adverse events related to follow-up procedures [2, 3]. It is also clear that more diagnostic procedures mean extra healthcare costs, but as stated before, diagnostic workup of ECFs can improve quality of life, which is a definite advantage for affected patients. Thus, our results seem to underline the fundamental importance of including a systematic analysis of ECFs in order to identify other causes that could potentially explain chest pain.
Furthermore, ours is the first study that found a significant positive association of female sex with clinically relevant ECFs. Our results suggest that women are more likely to have clinically relevant ECFs than men. A possible explanation for this observation is that women may have a lower pretest probability of CAD than men and consequently a higher probability of suffering from chest pain due to other, noncardiac causes [25]. An earlier study suggests that women with atypical chest pain and a clinical indication for ICA benefit from a reduction of minor procedural complications when undergoing CTA instead of ICA [26]. Our study suggests that the detection of ECFs which may potentially explain atypical chest pain is another clinical benefit for women.

It is already known that, in patients with atypical angina and a low to intermediate risk of CAD, performing CTA first defers ICA with no increase in long-term events, reduces minor procedural complications, and shortens the hospital stay compared to direct coronary angiography [21]. Our study has revealed an additional benefit of performing CTA in patients with atypical symptoms, namely the detection of ECFs which might constitute alternative causes of chest pain. Such incidental findings may contribute to the initiation of efficient diagnostic workup and therapy, thus eliminating the source of chest pain or even curing a potentially fatal malignancy.
Fig. 5 Fifty-six-year-old man with atypical load-independent chest pain not extending further. Diffuse idiopathic skeletal hyperostosis (DISH), also referred to as Forestier disease, was diagnosed and treated with analgesics and physical therapy, which contributed to chest pain relief

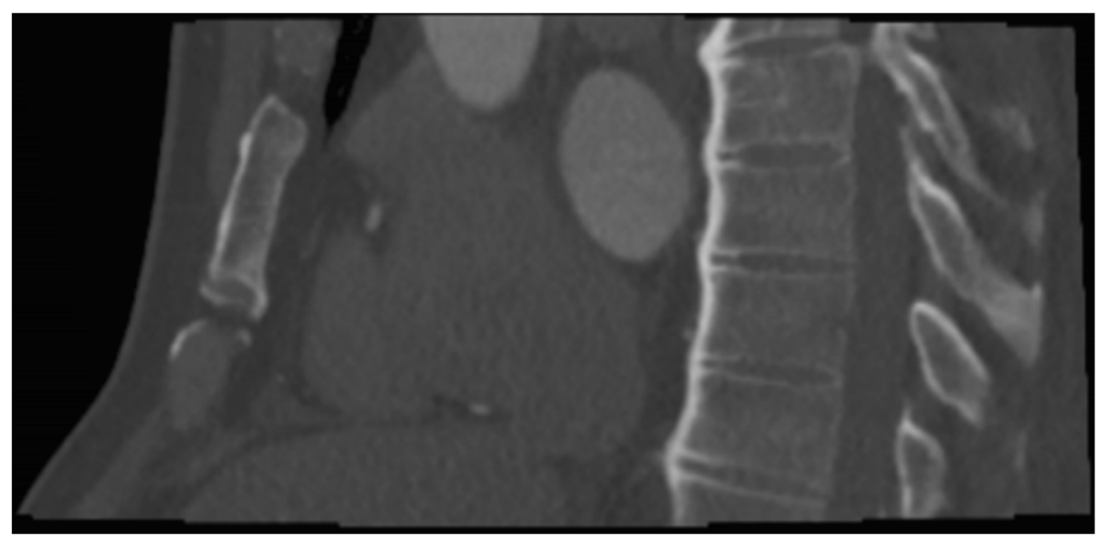




\section{Limitations}

Our study has several important limitations. First, due to a lack of other options, we used our subjective theoretical considerations to define the type and prevalence of ECFs classified as potentially explaining chest pain. Some follow-up examinations performed on an outpatient basis might not have been included if not consistently reported in the patient's follow-up questionnaire. Finally, the single-center design and the rather small number of patients included are important limitations. To obtain robust data on the clinical effectiveness and transferability to different clinical settings, investigation of a larger patient population and with longer follow-up and ideally in a multicenter trial is recommended. All of these requirements might be fulfilled by our ongoing multicenter randomized controlled DISCHARGE trial [27].

\section{Conclusions}

Our study is the first randomized comparison and shows that patients presenting with atypical angina or chest pain and a low to intermediate risk of CAD may have a twofold benefit from undergoing CTA instead of ICA: (1) detection of ECFs allowing early initiation of treatment or as potential explanation of patients' symptoms and (2) exclusion of obstructive CAD. Actionable clinically relevant ECFs detected by CTA affect patient management and therapy and may thus improve chest pain and consequently quality of life. Most of these ECFs would not have been detected by ICA.

Supplementary Information The online version contains supplementary material available at https://doi.org/10.1007/s00330-021-07967-x.

Funding Open Access funding enabled and organized by Projekt DEAL. Prof. Dr. Dewey has received grant support from the Heisenberg Program of the DFG (DE 1361/14-1).

\section{Declarations}

Guarantor The scientific guarantor of this publication is Prof. Dr. Marc Dewey

Conflict of interest The authors of this manuscript declare relationships with the following companies: Prof. Dr. Dewey has received grant support from the FP7 Program of the European Commission for the randomized multicenter DISCHARGE trial (603266-2, HEALTH-2012.2.4.-2). He also received grant support from German Research Foundation (DFG) in the Heisenberg Program (DE 1361/14-1), graduate program on quantitative biomedical imaging (BIOQIC, GRK 2260/1), for fractal analysis of myocardial perfusion (DE 1361/18-1), and the Priority Programme Radiomics for the investigation of coronary plaque and coronary flow (DE 1361/19-1 [428222922] and 20-1 [428223139] in SPP 2177/1). He also received funding from the Berlin University Alliance (GC_SC_PC 27) and from the Digital Health Accelerator of the Berlin Institute of Health. Prof. Dewey has received lecture fees from Canon, Guerbet. Prof. Dewey is European Society of Radiology (ESR) Research Chair (2019-2022) and the opinions expressed in this article are the author's own and do not represent the view of ESR. Per the guiding principles of ESR, the work as Research Chair is on a voluntary basis and only remuneration of travel expenses occurs. Prof. Dewey is also the editor of Cardiac CT, published by Springer Nature, and offers hands-on courses on CT imaging (www.ctkurs.de). Institutional master research agreements exist with Siemens, General Electric, Philips, and Canon. The terms of these arrangements are managed by the legal department of Charité-Universitätsmedizin Berlin. Professor Dewey holds a joint patent with Florian Michallek on dynamic perfusion analysis using fractal analysis (PCT/EP2016/071551). Other authors declared no conflicts of interest.

Statistics and biometry One of the authors has significant statistical expertise.

Informed consent Written informed consent was waived by the Institutional Review Board.

Ethical approval Institutional Review Board approval was obtained.

\author{
Methodology \\ - retrospective \\ - randomized controlled trial \\ - performed at one institution
}

Open Access This article is licensed under a Creative Commons Attribution 4.0 International License, which permits use, sharing, adaptation, distribution and reproduction in any medium or format, as long as you give appropriate credit to the original author(s) and the source, provide a link to the Creative Commons licence, and indicate if changes were made. The images or other third party material in this article are included in the article's Creative Commons licence, unless indicated otherwise in a credit line to the material. If material is not included in the article's Creative Commons licence and your intended use is not permitted by statutory regulation or exceeds the permitted use, you will need to obtain permission directly from the copyright holder. To view a copy of this licence, visit http://creativecommons.org/licenses/by/4.0/.

\section{References}

1. Karius P, Lembcke A, Sokolowski FC et al (2019) Extracardiac findings on coronary computed tomography angiography in patients without significant coronary artery disease. Eur Radiol 29: $1714-1723$

2. Williams MC, Hunter A, Shah ASV et al (2018) Impact of noncardiac findings in patients undergoing CT coronary angiography: a substudy of the Scottish computed tomography of the heart (SCOTHEART) trial. Eur Radiol 28:2639-2646

3. Bendix K, Jensen JM, Poulsen S, Mygind N, Norgaard BL (2011) Coronary dual source multi detector computed tomography in patients suspected of coronary artery disease: prevalence of incidental extra-cardiac findings. Eur J Radiol 80:109-114

4. Lee CI, Tsai EB, Sigal BM, Plevritis SK, Garber AM, Rubin GD (2010) Incidental extracardiac findings at coronary CT: clinical and economic impact. AJR Am J Roentgenol 194:1531-1538

5. Lazoura O, Vassiou K, Kanavou T, Vlychou M, Arvanitis DL, Fezoulidis IV (2010) Incidental non-cardiac findings of a coronary angiography with a 128 -slice multi-detector CT scanner: should we only concentrate on the heart? Korean J Radiol 11:60-68

6. Johnson KM, Dennis JM, Dowe DA (2010) Extracardiac findings on coronary CT angiograms: limited versus complete image review. AJR Am J Roentgenol 195:143-148 
7. Aglan I, Jodocy D, Hiehs S et al (2010) Clinical relevance and scope of accidental extracoronary findings in coronary computed tomography angiography: a cardiac versus thoracic FOV study. Eur J Radiol 74:166-174

8. Machaalany J, Yam Y, Ruddy TD et al (2009) Potential clinical and economic consequences of noncardiac incidental findings on cardiac computed tomography. J Am Coll Cardiol 54:1533-1541

9. Dewey M, Schnapauff D, Teige F, Hamm B (2007) Non-cardiac findings on coronary computed tomography and magnetic resonance imaging. Eur Radiol 17:2038-2043

10. Cademartiri F, Malago R, Belgrano M et al (2007) Spectrum of collateral findings in multislice CT coronary angiography. Radiol Med 112:937-948

11. Chia PL, Kaw G, Wansaicheong G, Ho KT (2009) Prevalence of non-cardiac findings in a large series of patients undergoing cardiac multi-detector computed tomography scans. Int J Cardiovasc Imaging 25:537-543

12. Gil BN, Ran K, Tamar G, Shmuell F, Eli A (2007) Prevalence of significant noncardiac findings on coronary multidetector computed tomography angiography in asymptomatic patients. J Comput Assist Tomogr 31:1-4

13. Greenberg-Wolff I, Uliel L, Goitein O et al (2008) Extra-cardiac findings on coronary computed tomography scanning. Isr Med Assoc J 10:806-808

14. Kim JW, Kang EY, Yong HS et al (2009) Incidental extracardiac findings at cardiac CT angiography: comparison of prevalence and clinical significance between precontrast low-dose whole thoracic scan and postcontrast retrospective ECG-gated cardiac scan. Int J Cardiovasc Imaging 25(Suppl 1):75-81

15. Onuma Y, Tanabe K, Nakazawa G et al (2006) Noncardiac findings in cardiac imaging with multidetector computed tomography. J Am Coll Cardiol 48:402-406

16. Koonce J, Schoepf JU, Nguyen SA, Northam MC, Ravenel JG (2009) Extra-cardiac findings at cardiac CT: experience with 1, 764 patients. Eur Radiol 19:570-576

17. Haller S, Kaiser C, Buser P, Bongartz G, Bremerich J (2006) Coronary artery imaging with contrast-enhanced MDCT: extracardiac findings. AJR Am J Roentgenol 187:105-110

18. Kawano Y, Tamura A, Goto Y, Shinozaki K, Zaizen H, Kadota J (2007) Incidental detection of cancers and other non-cardiac abnormalities on coronary multislice computed tomography. Am J Cardiol 99:1608-1609

19. Rumberger JA (2006) Noncardiac abnormalities in diagnostic cardiac computed tomography: within normal limits or we never looked! J Am Coll Cardiol 48:407-408

20. Budoff MJ, Fischer H, Gopal A (2006) Incidental findings with cardiac CT evaluation: should we read beyond the heart? Catheter Cardiovasc Interv 68:965-973

21. Dewey M, Rief M, Martus P et al (2016) Evaluation of computed tomography in patients with atypical angina or chest pain clinically referred for invasive coronary angiography: randomised controlled trial. BMJ 355:i5441

22. Diamond GA (1983) A clinically relevant classification of chest discomfort. J Am Coll Cardiol 1:574-575

23. Windecker S, Kolh P, Alfonso F et al (2014) 2014 ESC/EACTS Guidelines on myocardial revascularization: the Task Force on Myocardial Revascularization of the European Society of Cardiology (ESC) and the European Association for CardioThoracic Surgery (EACTS)Developed with the special contribution of the European Association of Percutaneous Cardiovascular Interventions (EAPCI). Eur Heart J 35:2541-2619

24. Karius P, Schuetz GM, Schlattmann P, Dewey M (2014) Extracardiac findings on coronary CT angiography: a systematic review. J Cardiovasc Comput Tomogr 8:174-182.e171-176

25. Regitz-Zagrosek V, Oertelt-Prigione S, Prescott E et al (2016) Gender in cardiovascular diseases: impact on clinical manifestations, management, and outcomes. Eur Heart J 37:24-34

26. Bosserdt M, Feger S, Rief M et al (2020) Performing computed tomography instead of invasive coronary angiography: sex effects in patients with suspected CAD. JACC Cardiovasc Imaging 13: 888-889

27. Napp AE, Haase R, Laule M et al (2017) Computed tomography versus invasive coronary angiography: design and methods of the pragmatic randomised multicentre DISCHARGE trial. Eur Radiol 27:2957-2968

Publisher's note Springer Nature remains neutral with regard to jurisdictional claims in published maps and institutional affiliations. 